\title{
escrito poŕn...
}

${ }^{1}$ Gabriela Vázquez-Díaz

Licenciada en Trabajo Social. Coordinadora del Proyecto VSSE.

Profesora de la Licenciatura en Trabajo Social en la Facultad de

Enfermería de la Universidad Autónoma de Yucatán.

\gabriela.vazquez@correo.uady.mx

(iD ORCID ID https://orcid.org/0000-0002-8491-981X

\section{${ }^{2}$ Amairani Ceh-Alvarado}

Licenciada en Trabajo Social. Colaboradora del Proyecto VSSE.

Profesora en la Facultad de Enfermería de la Universidad Autónoma de Yucatán.

\amairani.ceh@correo.uady.mx

(iD) ORCID ID https://orcid.org/0000-0002-4340-5724

\section{${ }^{3}$ Russel Carrillo-Puc}

Pasante de la Licenciatura en Trabajo Social. Prestador de Servicio

Social del Proyecto VSSE en la Facultad de Enfermería de la

Universidad Autónoma de Yucatán.

\russel.carrillo@correo.uady.mx

(D) ORCID ID https://orcid.org/0000-0003-4598-9441

Cómo citar / citation:

Vázquez-Díaz, G., Ceh-Alvarado, A., \& Carrillo-Puc, R. (2020). Sistematización de la intervención profesional durante el contexto de la COVID-19, para la valoración de la situación socioeconómica de jóvenes aspirantes a una institución educativa del sureste de México. Voces desde el Trabajo Social, 8(1), 16-39. https://doi.org/10.31919/voces.v8i1.217

Recibido / received: 31 de julio de 2020
Revisado / reviewed: 24 de noviembre de 2020
Aceptado / accepted: 8 de diciembre de 2020

Derechos de autoría / Copyright: (c) 2020 Vázquez-Díaz, G. et al. Este es un artículo de acceso abierto y distribuido bajo los términos de la licencia y políticas de Creative Commons Attribution 4.0 International License. 


\section{SISTEMATIZACIÓN DE LA INTERVENCIÓN PROFESIONAL DURANTE EL CONTEXTO DE LA COVID-19, PARA LA VALORACIÓN DE LA SITUACIÓN SOCIOECONÓMICA DE JÓVENES ASPIRANTES A UNA INSTITUCIÓN EDUCATIVA DEL SURESTE DE MÉXICO}

Gabriela Vázquez-Díaz ${ }^{1}$, Amairani Ceh-Alvarado 2 (D)

Russel Carrillo-Puc ${ }^{3}$

\section{Resumen}

fopenaccess PeEr-ReVIewed

El objetivo del artículo es sistematizar las funciones y habilidades que el trabajador social debe poseer para la intervención profesional desde medios alternos, e ilustrar los principales procesos implementados para la valoración socioeconómica de aspirantes a la educación media superior de la Unidad Académica Bachillerato con Interacción Comunitaria de una Universidad del Sureste mexicano, a raíz de la situación epidemiológica causada por la Covid-19, lo que lleva a realizar ajustes en la intervención considerando los lineamientos emitidos por las instancias internacionales y gubernamentales.

Por lo anterior, dicho documento se basa en la propuesta metodológica de Castañeda (2014) que consiste en: a) rescatar el contexto de la experiencia a); b) referentes teóricos; c) decisiones metodológicas; d) desarrollo del proceso de análisis con fines de la sistematización y e) reflexiones, aprendizajes y conclusiones del proceso.

Dentro de los resultados obtenidos de la sistematización se encontró que se requiere del compromiso profesional de los trabajadores sociales, rigurosidad metodológica y sus principios éticos, considerando que estos deben de estar presentes en toda intervención. Las funciones de relevancia durante el proyecto fueron la planificación, gestión, 
investigación y organización. Para realizar dichas acciones requiere de alinearse de aquellas medidas sanitarias, normativas institucionales, características de sus usuarios y, finalmente, de las competencias de sus profesionales que, a su vez exige la constante actualización y capacitación.

\section{Palabras claves}

sistematización, situación socioeconómica, Trabajo Social, jóvenes, educación.

\section{Sumario}

Introducción. Panorama diagnóstico de la educación en México. Una alternativa para el acceso a la educación. La contribución del trabajador social a la educación. Reconfigurando la intervención profesional ante la COVID-19. Propósitos de la sistematización de la experiencia. Fundamentación metodológica. Colectivos participantes de la experiencia. Técnicas y estrategias de recolección de información. Plan de análisis de la información. Procedimientos de validación. Consideraciones éticas. Resultados y hallazgos. Fase de planificación. Fase de ejecución. Fase de seguimiento y evaluación. El trabajador social y el contexto de la COVID-19. Discusión. Conclusiones. Referencias.

\section{[EN] SYSTEMATIZATION OF PROFESSIONAL INTERVENTION DURING THE CONTEXT OF COVID-19, FOR THE ASSESSMENT OF THE SOCIOECONOMIC SITUATION OF YOUNG APPLICANTS TO AN EDUCATIONAL INSTITUTION IN THE SOUTHEAST OF MEXICO}

\section{Abstract}

The objective of the article is to systematize the functions and skills that the social worker must possess for professional intervention from alternative means, and to illustrate the main processes implemented for the socioeconomic assessment of applicants to upper secondary education of the High School Academic Unit with Community Interaction of a University in the Southeast of the country, as a result of the epidemiological situation due to Covid-19, which leads to adjustments in the intervention considering the guidelines issued by international and governmental bodies. 
Therefore, this document is based on the methodological proposal of Castañeda (2014) that consists of: a) rescuing the context of the experience a); b) theoretical referents; c) methodological decisions; d) development of the analysis process for the purpose of systematization and e) reflections, learning and conclusions of the process.

Among the results obtained from the systematization, it was found that the professional commitment of social workers, methodological rigor and their ethical principles is required, considering that these must be present in every intervention. The relevant functions during the project were planning, management, research and organization. To carry out these actions, it requires alignment with those sanitary measures, institutional regulations, characteristics of its users and, finally, the competencies of its professionals, which, in turn, requires constant updating and training.

\section{Keywords}

systematization, socio-economic situation, social work, young people, education.

\section{Introducción}

Cuando se menciona que las nuevas generaciones son el futuro del país, se hace referencia a la oportunidad de cambio que representan, debido a que en la adolescencia, periodo comprendido de las edades de 10 a 19 años, y la juventud, extendida de los 15 a los 24 años de edad, las personas desarrollan sus capacidades para aprender, experimentar, utilizar el pensamiento crítico, expresar su libertad creativa y participar en procesos sociales, políticos, económicos, entre otros que atañen a nuestra sociedad (Organización Mundial de la Salud: OMS, 2020a;
Organización de las Naciones Unidas, 2019; Fondo de las Naciones Unidas para la Infancia, 2015).

En este sentido, a nivel nacional, la población que reside en México es de 119,938,473 habitantes, con base en la Encuesta Intercensal efectuada en el 2015 por el Instituto Nacional de Estadística y Geografía de México; ocupando los adolescentes y jóvenes el $27.32 \%$ de la población total. En el caso de Yucatán, el grupo antes señalado asciende a la cantidad de 572,487 habitantes del total de la población correspondiente a los 2,097,175; el cual representa el $27.29 \%$ de ésta (INEGI, 2015). 
Desde la disciplina de trabajo social, se contribuye en la educación de la población adolescente, al desarrollar el Proyecto Valoración de la Situación Socioeconómica de Aspirantes a la Educación Media Superior (Proyecto VSSE, en adelante), el cual busca ilustrar y esclarecer aspectos de la realidad social en el que se desenvuelven jóvenes residentes de la zona sur de Mérida, Yucatán, México; quienes aspiran a ingresar a la Unidad Académica Bachillerato con Interacción Comunitaria (UABIC, en adelante) de la Universidad Autónoma de Yucatán (UADY, en adelante), misma que busca apoyar a jóvenes que presentan condiciones socioeconómicas vulnerables (UABIC, S.F).

En el mes de marzo del 2020, en México inició la situación epidemiológica del SARS-COV2, virus que ocasiona la enfermedad conocida como la COVID-19; lo anterior trajo consigo como consecuencias las normativas de distanciamiento físico, suspensión de actividades que conlleven aglomeración, así como el uso de cubrebocas e implementación de la higiene de manos. Dentro del contexto estatal, se llevaron a cabo restricciones de movilidad, y nuevas formas de trabajo, lo cual demandó reconfigurar los procesos de intervención para el trabajador social dentro del Proyecto VSSE.
Ante esta reconfiguración de la intervención, se realizó la sistematización de la experiencia, mediante la propuesta metodológica para trabajo social en intervención social y sistematización de Castañeda (2014), quien define este proceso como "una estrategia de generación de conocimientos para el trabajo social latinoamericano, que aporta nuevas oportunidades de análisis y reflexión desde las dinámicas propias y particulares que caracterizan a la intervención social” (p.23).

\section{Panorama diagnóstico}

\section{de la educación en México}

La educación es un derecho humano fundamental en el que las personas adquieren, actualizan, completan y amplían sus conocimientos, capacidades, habilidades y aptitudes, de manera que puedan alcanzar su desarrollo humano integral, pero, a su vez contribuyan a la transformación y mejoramiento de la sociedad de la que forman parte, con un sentido de pertenencia social basado en la diversidad, equidad y solidaridad (Ley General de Educación, 2019).

En México, en el artículo $3^{\circ}$ de la Constitución Política de los Estados Unidos Mexicanos se indica que, dentro del Sistema Educativo Nacional, el nivel básico, está conformado por el prescolar, la primaria y secundaria, mientras que 
el nivel medio superior, comprende del bachillerato general, tecnológico y profesional técnico bachiller, mismos que deben de ser impartidos por el Estado -Federación, Estados, Ciudad de México y Municipios, de manera obligatoria.

Al respecto, la Dirección General de Planeación, Programación y Estadística Educativa (DGPPyEE, en adelante) de la Secretaría de Educación Pública (SEP) establece en el 2019 un modelo para analizar la eficacia del sistema educativo escolarizado a nivel nacional, en el cual se explica que de 100 estudiantes que inician la educación primaria, 27 abandonan temporal o definitivamente sus estudios durante su trayectoria por el nivel básico, de manera que, en la transición del nivel básico al medio superior, únicamente 66 estudiantes ingresan a un programa de bachillerato general, de los cuales 46 egresan de manera satisfactoria.

Ante este panorama, Vázquez (2008) señala que la educación media superior en México enfrenta cuatro graves problemas: la cobertura insuficiente, deserción escolar, la falta de calidad y esquemas de desigualdad. En estudios recientes dentro de este nivel educativo, el Instituto Nacional para la Evaluación de la Educación (2018), afirma una mejoría en el acceso y cobertura, sin embargo, la principal problemática es la permanencia de las y los estudiantes, la cual se vincula con elementos personales tales como: la falta de apoyo económico, las adicciones, situaciones de violencia y el poco interés de sus padres; así como con cuestiones institucionales dentro de las que mencionan las siguientes: profesores con poco interés, autoridades desvinculadas de la realidad de los estudiantes y pocos programas de apoyo a la educación superior.

En Yucatán, las problemáticas y necesidades relacionadas con la educación se visibilizan al encontrar que el grado promedio de escolaridad para el ciclo escolar 2018-2019, es de 9.2 años, es decir, un nivel de estudios correspondiente a la primaria y secundaria completa. Además, se identifica que solamente el $67.6 \%$ de los estudiantes que ingresan culminan de manera satisfactoria nivel medio superior (DGPPyEE, 2019). Siendo así que existe un problema de acceso y terminación del nivel medio superior.

\section{Una alternativa para \\ el acceso a la educación}

Como se ha descrito, la principal problemática identificada se centra en el abandono y reprobación escolar en la educación media superior, lo que también repercute en el egreso y continuación de estudios de 
las y los estudiantes. Desde una perspectiva ecosistémica, existen aspectos del contexto social en el que se desenvuelve la persona, que influyen en su trayectoria escolar, sobresaliendo la falta de recursos económicos, problemas de salud, situación económica, laboral y educativa de los padres o familia, motivación, entre otros.

Asimismo, de manera estructural, se identifica que, en Mérida, Yucatán, a partir de la segregación urbana y distribución espacial que se ha desarrollado en las últimas décadas, existe una marcada polarización entre las zonas norte y sur de la ciudad, la primera es caracterizada por ser para grupos con mayor poder adquisitivo y en la segunda se focalizan problemáticas como la marginación, el desempleo, pobreza y exclusión social (García, Oliva y Ortiz, 2012; Comisión Nacional para Prevenir y Erradicar la Violencia contra las Mujeres: CONAVIM, 2009).

García y Ruiz (2011) señalan que, en lo relativo al aspecto social, las problemáticas latentes en la zona sur son las toxicomanías, la pobreza, la inactividad juvenil relacionada con aquellos que no estudian ni trabajan; el desempleo, y el retraso escolar, el vandalismo, pandillerismo, delincuencia, y violencia. En el aspecto económico, el desempleo, los empleos informales, baja percepción de ingresos económicos, lo anterior ligado con el aspecto urbano, en el que se describe la falta de instituciones educativas en la zona, infraestructura como pavimentación de calles y alumbrado público, espacios de recreación, hacinamiento, falta de servicios públicos, entre otros. Ante este panorama, García, Oliva y Ortiz (2012) enfatizan en la intervención estatal y municipal para una mejora del ingreso, así como mayor inversión en educación, sanidad y vivienda con el fin de reducir las patologías sociales como la drogadicción, delincuencia, y violencia.

En el 2009, surge la UABIC como parte de las instituciones educativas del nivel medio superior de la UADY, en respuesta a las necesidades sociales de la región, el crecimiento demográfico y la falta de espacios físicos para el número de solicitantes. Esta oferta educativa, está dirigido a las personas de comunidades del territorio yucateco, cuyas condiciones socioeconómicas de vulnerabilidad, dificulta su preparación académica en el nivel medio superior (UABIC, S.F.).

Ante los fines planteados y al incremento del número de aspirantes, se identifica que una problemática existente es focalizar a las y los aspirantes que cumplan con el perfil al cual va dirigido el Programa Educativo antes señalado, es decir, identificar en la población adolescente 
situaciones de vulnerabilidad, así como la capacidad de concluir con éxito su preparación académica, esto a través de la obtención de un puntaje determinado en el Examen Nacional de Ingreso a la Educación Media Superior (EXANI- I).

\section{La contribución del trabajador social a la educación}

De acuerdo con Galeana (2005), las funciones que el trabajador social desempeña en el ámbito educativo son la investigación, programación, educación, orientación y asistencia, mismas que se reflejan en acciones como la identificación y caracterización de factores económicos-sociales y culturales que intervienen en los procesos de reprobación y deserción escolar, realización de estudios sociales que sirvan de base para la asignación de recursos y apoyo educativo, además de integrarse en equipos interdisciplinarios de carácter socio-pedagógico, entre otras acciones.

Con fundamento en lo anterior, en el año 2018, el Programa de la Licenciatura en Trabajo Social de la UADY, colaboró con un grupo multidisciplinario de expertos (as) en el área de Estadística, Actuaría, Psicología, Educación para la elaboración de una encuesta socioeconómica en línea, que permita la identificación de situaciones de vulnerabilidad de manera estadística. Este instrumento, forma parte del proceso inicial de admisión, el cual es complementado con la intervención del profesional en trabajo social.

Para la intervención profesional se diseñó el Proyecto VSSE con fundamento en la perspectiva ecológica y sistémica, mismo que se encuentra adscrito al proceso de ingreso al bachillerato. Su propósito principal es que a partir de la aplicación de una serie de instrumentos y técnicas se ilustre y esclarezcan aspectos de la situación socioeconómica en la que se desenvuelve tanto el aspirante como su familia para dictaminar si cumple con las condiciones de vulnerabilidad para ingresar a la UABIC, no obstante, en caso de no cumplir con los criterios establecidos, se le invita a participar en el proceso de ingreso a los programas de bachillerato ofertados tanto por la Universidad1 como por otras Instituciones Educativas del Estado.

\section{Reconfigurando la intervención profesional ante la COVID-19}

La COVID-19 es la enfermedad infecciosa causada por el coronavirus que se ha descubierto más recientemente en Wuhan, China. El 11 de marzo del 2020 fue declarada por la OMS como pandemia luego de identificarse que el número de casos de COVID-19 fuera de China se ha multiplicado por 13, y el número de países afectados se ha triplicado (OMS, 2020b). 
Con respecto a la situación epidemiológica en México, se ha identificado que hasta el día 30 de julio de 2020, existen 416, 179 casos confirmados, y 46, 000 defunciones. En el caso de Yucatán la prevalencia de casos confirmados es de 9, 438 y 843 defunciones. Asimismo, Mérida, es uno de los municipios con mayor número de casos reportados, al indicarse la presencia de 5, 344 casos confirmados y 434 defunciones (Dirección General de Epidemiologia, 2020).

Ante tal panorama, y bajo la premisa que todas las personas son vulnerables a contagio de la COVID19, se implementaron medidas para la interacción social, entre las que destaca el distanciamiento, lo cual demandó el ajuste en las formas de cumplir con actividades laborales desde medios alternos. En este sentido, desde el plano laboral, surgió un nuevo esquema virtual, en el que se ha adaptado el ejercicio profesional a través de las distintas tecnologías de la comunicación, para salvaguardar y procurar la salud de las personas.

Es así como la contingencia sanitaria y las normas emitidas para la interacción social representan uno de los principales retos para los que trabajadores sociales, debido a que limita la intervención presencial. Por tal motivo, se requiere generar nuevas alternativas que se apeguen a las indicaciones sanitarias $y$ medidas de prevención, dentro del contexto de la COVID-19 y, que, a su vez, mantenga la rigurosidad metodológica de la disciplina, conllevando así a reconfigurar los procesos de intervención del Proyecto VSSE 2020.

\section{Propósitos de la sistematización de la experiencia}

La transición hacia una intervención desde medios alternos a consecuencia de las medidas sanitarias implementadas por la pandemiarequiere de una redefinición de las formas de hacer el trabajo social, al que se ha construido en otros contextos previo a la COVID19; siendo la sistematización el medio favorable.

Al respecto, Jara (2013) conceptualiza a la sistematización como aquella interpretación crítica de una $o$ varias experiencias que, a partir de su ordenamiento y reconstrucción, descubre o explica la lógica del proceso vivido en ellas. Asimismo, produce conocimientos y aprendizajes significativos que posibilitan la comprensión teórica de la experiencia y orientarlas hacia el futuro con una perspectiva transformadora.

En este sentido, Castañeda (2014) señala que lo deseable para sistematizar, es acotar en forma operativa aspectos específicos de 
la experiencia para potenciar la profundidad en el análisis, además, Castro (2016) añade que en esta fase se debe determinar qué parte de la experiencia se va a recuperar, y los periodos que se quieren sistematizar.

A partir de señalado con anterioridad, se plantea como objetivos para la sistematización del Proyecto VSSE, determinar las funciones y habilidades que el trabajador social debe poseer para la intervención profesional desde medios alternos, e ilustrar los principales procesos implementados para la valoración socioeconómica de aspirantes a la educación media superior a partir de la efectuación de entrevistas telefónicas.

La duración de este proyecto de manera intermitente fue de seis meses, parafines delasistematización se retoma lo realizado a partir de la suspensión de actividades en Yucatán, México, del 16 de marzo hasta la culminación de la evaluación del mismo, en el mes de julio de 2020.

El proyecto se constituyó en las siguientes fases (Ver figura 1), que serán abordadas más adelante.

\section{Figura 1. Fases del proyecto VSSE-2020}

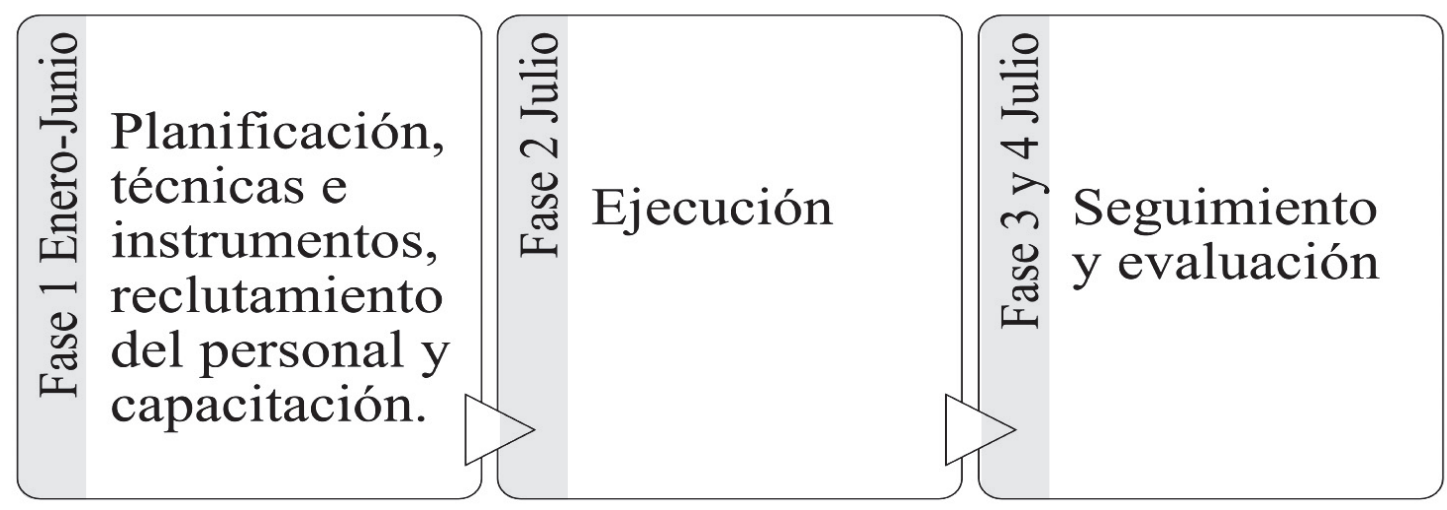

Fuente: Elaboración propia, 2020.

\section{Fundamentación metodológica}

La generación de conocimientos es un aspecto que la sistematización produce, a partir de la experiencia en la intervención profesional (Castañeda, 2015), por lo tanto, precisa definir y rescatar elementos relevantes de la misma. En este sentido en la Escuela de Trabajo Social de la Universidad de Valparaíso propone una secuencia para la sistematización que consiste en la siguiente: a) Contextos de la experiencia, b) Referentes teóricos, c) Decisiones metodológicas, d) Desarrollo del proceso de análisis con fines de 
la sistematización y e) Reflexiones, aprendizajes y conclusiones del proceso (Castañeda, 2015, p.27-29).

Por lo anterior, se retoma dicho proceso para fundamentar la experiencia vivida en el Proyecto VSSE en el 2020, considerando que para la muestra en la fase de "Ejecución de las entrevistas telefónicas y elaboración de informes sociales" se utilizó el programa Epi info Versión 7, obteniendo 226 aspirantes a partir de una población de 548. Cabe señalar, que fueron cinco visitas domiciliaras efectuadas con toda la protección y seguridad señalada ante la pandemia de la COVID-19, y los demás casos (221) se realizaron entrevistas vía telefónica para corroborar la situación reportada en línea de manera previa, con base al proceso de ingreso al Bachillerato 2020.

\section{Colectivos participantes}

\section{de la experiencia}

Los participantes de este proyecto de manera interna representan al grupo de 17 trabajadores sociales que efectuaron las entrevistas o visitas domiciliarias, así como el equipo encargado de la logística de este (8 personas), quienes fungieron, algunos, como coordinadores y/o revisores, encargados de dar continuidad y seguimiento a las actividades que se derivan del proyecto y ocasionalmente realizaban entrevistas y elaboración de informes.
Los profesionales antes señalados tienen el siguiente perfil: a) conocimiento de técnicas e instrumentos de Trabajo Social; b) habilidad en investigación social, en la aplicación de entrevistas y realización de estudios socioeconómicos; c) experiencia en investigación (trabajo) de campo y en redacción de informes y diagnósticos sociales; d) actitud de responsabilidad y compromiso disponibilidad de horario.

Asimismo, de manera externa se encuentra la institución a la que se proporcionó el servicio; sin embargo, para efectos de este documento y con base al objetivo de éste, solamente se incluye al colectivo participante de manera interna, ya que se refiere al proceso que se experimentó con aquellos agentes participantes.

\section{Técnicas y estrategias de recolección de información}

La sistematización de la práctica se caracteriza por recuperar la experiencia vivida durante la intervención social en los distintos escenarios en los que los trabajadores sociales se desempeñan. En este sentido, Castañeda (2015) indica que la elección de las técnicas y estrategias para la recolección de información deben estar en función de los fines planteados para la sistematización.

En consecuencia, se inició el proceso con la revisión documental 
de las distintas herramientas $e$ instrumentos empleados durante la intervención, como son fotografías, vídeos, notas de campo, informes sociales, bitácoras, cuestionarios y reportes, los cuales de acuerdo con Castañeda (2014) resultan fuentes de información que permiten reescribir la experiencia desde sus propios códigos y exponerla con mayor precisión en el análisis. Acto seguido, se realizaron reuniones grupales para profundizar en lo obtenido de la revisión, de tal manera que, la aportación de cada integrante favoreció la reflexión profesional, y la integración de la información en un informe final de actividades.

\section{Plan de análisis de la información}

Se inició el análisis desde un nivel descriptivo en el que a partir de la evidencia y la reflexión profesional se reconstruyólapráctica, categorizando la información obtenida en función de las fases por las que transitó el proyecto, lo anterior con el objetivo de identificar sus interrelaciones, las técnicas, instrumentos, herramientas, funciones, aspectos teóricos y metodológicos de la intervención. Además de precisar las fortalezas, áreas de oportunidad y retos enfrentados por los trabajadores sociales durante su ejercicio profesional; esta etapa se apoyó de la elaboración de estructuras de secuencia, tablas, figuras, entre otros, que permitieron la síntesis y ordenamiento de la información. Finalmente, se ilustraron los procesos claves de la intervención social, es decir, se integraron las reflexiones, aprendizajes, y conclusiones obtenidas desde la experiencia de la práctica.

\section{Procedimientos de validación}

La validación utilizada en este proceso de sistematización fue la denominada validación por expertos, en donde profesionales en el área revisan y externan su punto de vista con respecto al proceso llevado a cabo. En este caso participaron tres profesionales del área social (Trabajo Social y Psicología) y otros de manera inmediata, los y las trabajadores sociales involucrados en el proyecto a través de un instrumento de evaluación de este.

\section{Consideraciones éticas}

El desarrollo de la sistematización del Proyecto VSSE, se basó de la Declaración Global de Principios Éticos del Trabajo Social propuesto por la Federación Internacional de Trabajadores Sociales (FITS, 2018), en este sentido, como profesionales de la disciplina, se respetó la confidencialidad y la privacidad de las personas, la aplicación de los principios éticos en la práctica del trabajo social e investigación mediante el uso de la tecnología digital y las redes sociales. 
Lo expresado anteriormente, se refleja en la protección de los datos personales de los actores involucrados en el desarrollo del proyecto, así como en el análisis de la información, para el cual únicamente se consideraron los procedimientos, técnicas, instrumentos, herramientas, aspectos teórico-conceptuales y metodológicos, implementados para la intervención profesional.

\section{Resultados y hallazgos}

El trabajo social es una profesión que interviene directamente en la realidad social, misma que está sujeta a los sucesos emergentes que ocurren en el contexto, este tipo de acontecimientos pueden ser inesperados y de los que no se tiene un registro previo en los últimos años, motivo por el cual la generación estrategias para atender las emergentes necesidades y sus efectos colaterales son pieza clave para la intervención.

Como se mencionó en un inicio, el confinamiento social ha sido uno de los causantes para restringir la intervención de contacto directo con los usuarios, puesto que el proyecto se alinea a las indicaciones estales de salud y normativas institucionales. Lo anterior, es una razón para reconfigurar el quehacer profesional de Trabajo Social, ocasionando que, modifique sus técnicas de investiga- ción e intervención, demuestre sus habilidades y actitudes profesionales, pero a la par, mantenga su ética profesional y su rigurosidad metodológica que la caracteriza de manera disciplinar.

Como idea inicial, se plantea realizar una visita domiciliaria que incluye una entrevista al usuario, entrevistas colaterales, y finaliza con la redacción de un informe social en donde se añade una descripción de la situación encontrada y una opinión profesional, mediante la cual se identifica las vulnerabilidades del aspirante, sin embargo, las experiencias que se plasman en el presente artículo se derivan de la participación en el Proyecto VSSE durante el contexto de la COVID-19.

La sistematización de la intervención profesional se divide según las fases del proyecto, teniendo como principales: planificación, ejecución, seguimiento y evaluación; se retoman las modificaciones que se realizaron en cada una, así como los aprendizajes obtenidos, para la mejora de próximas aplicaciones del proyecto y fungir como referente a otros profesionales.

\section{Fase de planificación}

Para comenzar, la planificación del proyecto incluye la elección de las técnicas a emplear, sus instrumentos, capacitación al personal y actividades 
de logística. La técnica principal del proyecto, según promociones de años pasados, son las visitas domiciliarias, no obstante, a partir de los efectos colaterales del contexto de la COVID-19, se requirió realizar adaptaciones metodológicas para salvaguardar la salud de los usuarios y colaboradores del proyecto.

El proceso de adaptación durante la fase de planificación se derivó de una serie de elementos a considerar, los cuales permitieron valorar que las propuestas de modificaciones ante el contexto emergente sean viables, pertinentes y factibles. Las pautas para considerar fueron las siguientes:

a. el objetivo principal de la intervención, esto se refiere al propósito del proyecto donde se está inmerso, ya que únicamente se modificaron las técnicas e instrumentos a emplear.

b. las tecnologías de la información y comunicación, resulta importante incluir nuevas formas de comunicación en entornos virtuales que ofrezcan la oportunidad de interactuar y establecer una vía de comunicación de dos tipos, entre trabajadores sociales y trabajador social-usuario. c. la capacidad institucional, esto se refiere a los recursos humanos, técnicos, materiales, económicos e informativos, con los que cuenta la institución donde se está inmerso, para dar respuestas a las propuestas o sugerencias, garantizando que sean viables y factibles.

d. las competencias de los profesionales, es importante identificar los conocimientos, habilidades y actitudes de los trabajadores sociales que efectuarán la intervención, ya que, de este modo, se analiza si se pueden llevar a cabo las modificaciones y adecuaciones previstas.

Basado en los criterios anteriores, en la figura 2 se presentan los elementos aplicados en el proyecto. Así entonces, a partir de un análisis de la viabilidad, pertinencia y factibilidad, se optó por llevar a cabo la aplicación de entrevistas semi-estructuradas mediante la vía telefónica en lugar de visitas domiciliarias, así como realizar los procesos de capacitación y reuniones de trabajo mediante plataformas educativas virtuales. 
Figura 2. Objetivo y elementos del proyecto VSSE-2020

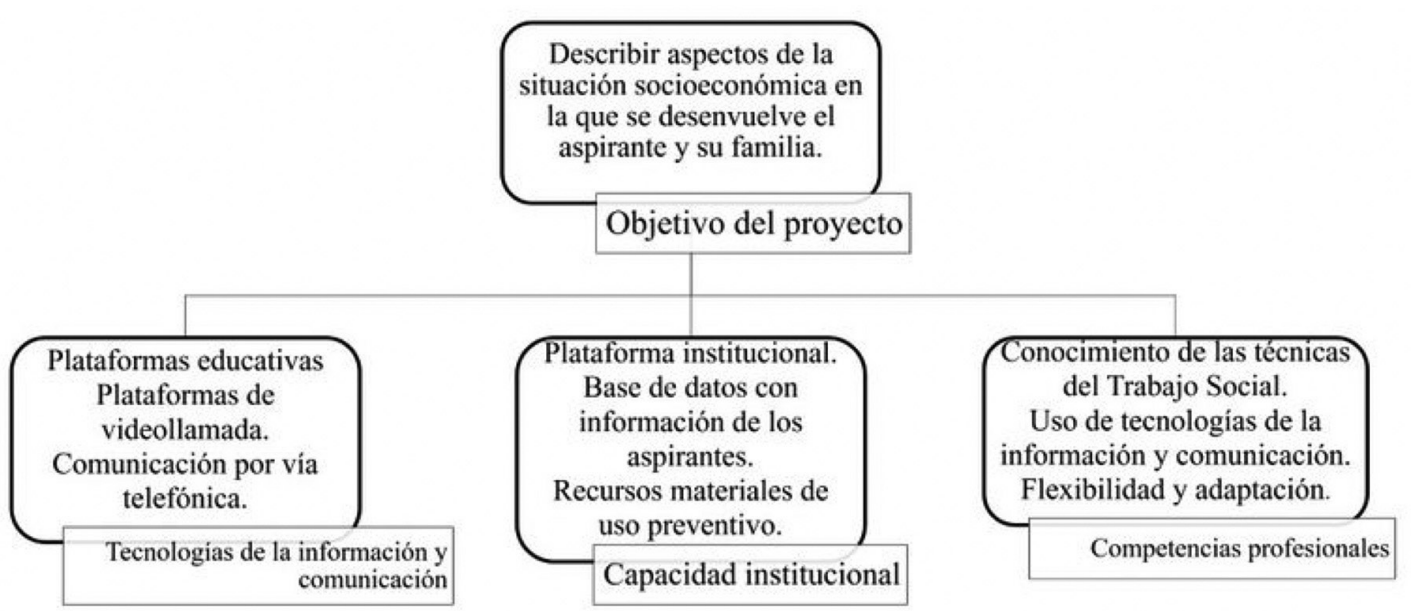

Fuente: Elaboración propia, 2020.

\section{Fase de ejecución}

Continuando con la segunda fase, durante la ejecución de este proyecto se identifica que, si bien las condiciones sanitarias de la actualidad son una limitante para el contacto directo con los usuarios, esto no quiere decir que dentro de las vías de comunicación alternas se pierda el humanismo y la ética profesional. Basado en lo anterior, fue importante cuidar los principios de confidencialidad, beneficencia y autonomía.

En el primer contacto se emitió un saludo inicial y presentación del profesional con el fin de generar un ambiente de confianza con el usuario; seguidamente, se explicó en qué consiste el proceso de entrevista, para asegurar la autonomía de participación; y finalmente, se señaló que los datos únicamente son para los fines propios del proyecto, asegurando así, la confidencialidad de estos.

Asimismo, se rectifica que la adaptación al contexto actual no debe modificar la rigurosidad metodológica de la actuación profesional durante su ejecución. En este caso, se requiere de una validez de la información, misma que se procuró por medio de la triangulación de datos, teniendo como fuentes principales: (a) entrevistas con el tutor del aspirante, (b) datos de la encuesta socioeconómica en línea, (c) documentos comprobatorios (d) entrevistas de referencias, familiares, amigos, y/o centros de trabajo, (e) páginas gubernamentales y oficiales de programas sociales.

Considerando las estrategias aplicadas por las y los trabajadores sociales se visualiza que, en la 
actualidad, existen diferentes medios por los cuales se pueden obtener información confiable acerca de usuarios (as), sin embargo, pone en una disyuntiva la privacidad de los datos y el uso de éstos; por lo que deben de ser única y exclusivamente para los fines propios del proyecto, además de salvaguardar los datos de otras personas que no están involucradas.
Durante la ejecución de las entrevistas semi-estructuradas y la redacción de informes sociales, se identificaron nuevos conocimientos, habilidades y actitudes que los trabajadores sociales deben de tener para su implicación dentro de la realidad social o contexto de la COVID-19; este perfil se resume la Tabla 1.

\section{Tabla 1. Perfil del profesional de Trabajo Social en el proyecto VSSE}

\section{Conocimientos}

- Qué es el COVID-19, vías de contagio, medidas preventivas.

- Actualizaciones acerca del contexto económico y social, de las variables que intervienen en el proyecto.

- Técnicas del Trabajo Social.

- Elementos teóricos para identificar nuevas vulnerabilidades.

\section{Habilidades}

- Rapport

- Generar un ambiente de confianza.

- Escucha activa. problemas.

- Uso de las tecnologías de • Ética la información y profesional. comunicación. - Humanismo.
En cuanto a los conocimientos, se identificó que una de ellas es saber qué es la COVID-19 y sus medidas preventivas, debido a que en cinco ocasiones se tuvo la necesidad de acudir a visitas domiciliarias por no lograr contactar a los usuarios. Así entonces, se salvaguarda la salud de los participantes y de los colaboradores.
De igual manera, para la elaboración de informes sociales se requirió de la revisión de las actualizaciones de los parámetros socioeconómicos de diversas instituciones, como por ejemplo, la Línea de Pobreza por Ingreso del Consejo Nacional de Evaluación de la Política de Desarrollo Social (CONEVAL), las restricciones labo- 
rales, las condiciones de empleo, nuevos programas sociales, entre otros elementos, que han sido consecuencia del confinamiento social en el aspecto socioeconómico, y han tenido un impacto directo en los contextos de los aspirantes.

Referente a las habilidades, resalta el uso de las tecnologías de la información y comunicación, debido a que fueron el medio imprescindible para efectuar las entrevistas, la redacción y entrega de informes, además de ser una herramienta para la triangulación de datos. Aunado a lo anterior, para cuidar que la información sea de calidad fue importante contar con las habilidades de rapport y escucha activa.

Continuando con la descripción del perfil, resaltaron las actitudes propias de la disciplina, entre las que destacan la creatividad, adaptación y flexibilidad, puesto que el proyecto fue modificado y se tuvieron obstáculos en la ubicación de los tutores, obtención de documentos comprobatorios, según los casos asignados. Igualmente, fue importante la empatía de los profesionales durante la intervención, porque se estuvo en contacto con usuarios que también viven una "nueva realidad".

\section{Fase de seguimiento y evaluación}

La tercera fase dentro del proyecto fue el seguimiento, actividad prescindible para la intervención, debido a que facilita la identificación de cómo se están presentando las acciones, si se está cumpliendo con el objetivo, o por el contrario generar las adecuaciones necesarias. Aplicado en la práctica, se realizó un seguimiento de entrevistas, elaboración de informes sociales, y principalmente, la atención de dudas directas por parte del equipo de logística; lo anterior favoreció identificar aquellos casos en los cuales no se contactó a los tutores para efectuar las entrevistas, para proceder medios alternos de comunicación, y en su caso, visitas domiciliarias.

Por último, la evaluación fue por medio de una encuesta en línea, con preguntas enfocadas al proyecto, desde la capacitación, comunicación con el equipo de logística, instrumentos empleados, fortalezas, áreas de oportunidad, hasta propuestas de mejora. En conjunto con lo anterior, se elaboró un informe general de las actividades, en el cual se retoma la valoración objetiva del proceso en función de los resultados obtenidos.

A partir de los instrumentos mencionados, se facilita el proceso de sistematización, en el cual se 
rescatan aprendizajes de la práctica. Es preciso resaltar que los recursos tecnológicos fueron adecuados para la capacitación y la entrega de productos, obteniendo que la comunicación no se limita a un acercamiento cara a cara, sino que se remite a la escucha activa y al asertividad.

Con respecto a las fortalezas del proyecto se identifica que, desde la planificación del proyecto, estuvieron presentes las habilidades y actitudes del trabajador social, tales como: flexibilidad, adaptación, el uso de las tecnologías de comunicación, el trabajo en equipo y la creatividad ante la solución de problemas. De manera resumida se destacó la organización en los tiempos, el acompañamiento en los procesos, la atención oportuna de dudas o problemáticas, la comunicación constante, y trabajo colaborativo.

Si bien se encontraron fortalezas, también se hallaron áreas de oportunidad como el tiempo de duración del proyecto, ya que este se dirige y apega a las normativas institucionales de la universidad. Asimismo, se propone integrar al instrumento utilizado un apartado de egresos de manera más desglosada, debido a que las familias de los aspirantes presentan mayores gastos y viven diferentes situaciones que, aunado a la pandemia de la actualidad, las posicionan en situaciones de riesgo y aportan a la vulnerabilidad acumulada.

A partir de la experiencia del proyecto, se identifican retos que surgen a partir de los nuevos contextos, entre los que se encuentran: garantizar que el uso de las tecnologías no excluya a ningún usuario o que esta alternativa sea una limitante para ellos; procurar que la comunicación sea directa con los involucrados para que sea de manera efectiva; asegurar la confidencialidad de los datos proporcionados y que no se den a conocer o permanezcan en sitios indebidos; mantener la rigurosidad metodológica del trabajo social, con respecto a las técnicas, instrumentos, procesos y consideraciones éticas, y por último, generar estrategias efectivas.

\section{El trabajador social y el contexto de la COVID-19}

Los conocimientos empíricos no solamente recaen en la intervención que tiene que ser sistematizada, sino también en las nuevas realidades de las personas y en el análisis de los cambios sociales que se obtuvieron. De manera general, se visualizó que el confinamiento social añade mayores vulnerabilidades a las personas, ya que trae consigo cambios en las actividades laborales y remuneraciones económicas, 
teniendo así una variación en los resultados.

A partir de la descripción de las fases del proyecto, se rectifica que el trabajador social dentro de su compromiso profesional se adecua a los contextos donde está inmerso, aplicando sus conocimientos, habilidades y actitudes. Es por todo lo anterior, que se identifica que ante la nueva normalidad y las actividades profesionales durante la contingencia sanitaria cumplen con las siguientes funciones: a) Planeación, recae en considerar las nuevas condiciones del contexto, retomando medios alternativos, la capacidad institucional, competencias profesionales, y principalmente, la pertinencia y viabilidad de sus acciones a realizar. b) Gestión, es de suma importancia, puesto que de acuerdo con su planeación se van a requerir recursos humanos, técnicos, materiales y económicos, por lo cual deberá ser un intermediario. c) Investigación, esta puede ser desde la parte documental de actualizaciones del contexto, parámetros, hasta una de tipo social, en donde aplicará sus técnicas e instrumentos e incluirá sus procesos de validez de información. d) Organización, debido a que se está en una adaptación de las actividades, con nuevos protocolos y normativas.

Aunado a lo anterior, el trabajador social deberá de tener en cuenta que el empleo de los recursos tecnológicos como medio de comunicación se ha posicionado como la principal alternativa ante la contingencia sanitaria, por lo que se requiere su compromiso de actualización y manejo de ellas, con el fin de cumplir con sus objetivos profesionales.

Se hace hincapié en la necesidad de la creatividad ante la solución de problemas, considerando los recursos con los que cuenta, y tener una flexibilidad ante los cambios, ya que no dependen directamente de decisiones individualizadas, sino que también de condiciones sanitarias, institucionales y reglamentarias. Como último punto, la empatía entra como una actitud de suma importancia ante la interacción con los usuarios, puesto que para ellos también pueden ser nuevos los procesos y ambientes en los que tenemos contacto, estando aún en adaptación.

\section{Discusión}

Como parte de contraste de los resultados obtenidosy de las recientes bibliografías que han surgido, se coincide con Salcedo (2020), cuando menciona que en este contexto se requieren de trabajadores sociales comprometidos, competentes, creativos y capaces, ya que son tiempos complejos con cambios radicales donde los profesionales tienen que asumir los retos que 
se presentan. La idea anterior, representa los criterios considerados para la adecuación del proyecto, visualizando aquellos conocimientos, habilidades y actitudes que se deben de tener para cumplir con el compromiso adquirido, puesto que de ese informe social depende el futuro de las familias.

Asimismo, Varea (2020) resalta que la intervención en la actualidad debe de partir desde una adecuada planificación de las acciones, creando redes de atención, coordinación entre los profesionales, identificar recursos que faciliten la intervención y mantener una adecuada comunicación; esto se vio implicado durante el proyecto desde un análisis de diversos elementos para una mejor actuación profesional, motivo por el cual se coincide que la planificación queda como una fase determinante de la que dependerá el cumplimiento de los objetivos profesionales.

\section{Conclusiones}

La figura del Trabajador Social en el ámbito educativo permite detectar y atender tanto problemáticas como necesidades con incidencia en la trayectoria académica de estudiantes, mediante las múltiples funciones que se desarrollan; es así como en la recuperación teórica de la experiencia se reconoce que la investigación social es una función que permite identificar la vulnerabilidad del entorno familiar en el que aspirantes a bachillerato se desenvuelven; contribuyendo de esta manera en el cumplimiento del objetivo planteado por la institución y en el acceso a oportunidades de estudio.

Aunado a lo antes descrito, la sistematización de la práctica es reconocida como parte fundamental de los procesos reflexivos de la intervención, debido a que generan aprendizajes y nuevos elementos teóricos. Lo anterior, resulta relevante para el colectivo de Trabajadores Sociales, ya que permite orientar y fundamentar el actuar profesional, además de una actualización continua de los procesos de intervención a partir de los cambios que acontecen en el contexto inmediato, como es el caso del escenario dejado por el virus SARS-CoV-2, en el que demanda un reajuste y repensar del quehacer del trabajador social.

Como principales reflexiones de la implementación del proyecto VSSE ante el contexto por la COVID-19 a la que la población mundial se enfrenta, se reconoce el compromiso profesional de los trabajadores sociales para realizar las modificaciones y ajustes necesarios a su intervención, conservando su rigurosidad metodológica y principios éticos, que deben considerarse en todo momento. Asimismo, se 
identificó la necesidad de reforzar el uso y la protección de información personal de todos los participantes en el proyecto. Para cumplir con lo anterior, es necesario alinearse a las medidas sanitarias, normativas e institucionales vigentes, además de las características de la población objetivo y, de las competencias de sus profesionales quienes deben actualizarse y capacitarse constantemente.

Finalmente, como recomendaciones, se sugiere que para el actuar profesional ante la COVID19, se consideren como aspectos principales, el objetivo de la intervención, mismo que debe ser concreto y acorde a la realidad social a la que se oriente, las tecnologías de la información y comunicación, la capacidad institucional, así como las competencias profesionales. Sumando a lo anterior, es necesario una planeación y programación, la delimitación de las funciones y actividades que cada miembro del colectivo participante debe cumplir. De igual manera, es preciso indagar acerca de las vulnerabilidades que emergen como consecuencia del virus SARS-CoV-2, además de otros aspectos que se consideren pertinentes; lo anterior realizando investigaciones documentales $y$ sistematizaciones de experiencias previas, o similares.

\section{Agradecimiento:}

Agradecimiento a la Dirección General de Desarrollo Académico y la Coordinación del Sistema de Educación Media Superior de la Universidad Autónoma de Yucatán, a la Facultad de Enfermería, a los profesionales involucrados y a los participantes del proyecto.

\section{Declaración de conflicto de intereses:}

Las personas autoras declararon que no hay conflictos de interés potenciales con respecto a la investigación, autoría o publicación en este artículo.

\section{Declaración sobre lenguaje inclusivo:}

En esta investigación se utilizará, principalmente, el género gramatical masculino para referirse a diversas identidades humanas o colectividades (por ejemplo, trabajadoras y trabajadores sociales) sin que esto suponga un lenguaje sexista y excluyente. 


\section{Referencias}

Castañeda, P. (2014). Propuestas metodológicas para Trabajo Social en Intervención Social y Sistematización: Cuaderno Metodológico. http://www.ts.ucr.ac.cr/ binarios/libros/libros-000043.pdf

Castañeda, P. (2015).

Sistematización y generación de conocimientos en trabajo social. Aportes metodológicos a la formación profesional. Alternativas Cuadernos de trabajo social, 1 (22), 23-32.

Castro, M. (2016). Sistematización en Trabajo Social: un proceso de construcción del conocimiento entre la práctica y la teoría.

Editorial de Lito-Grapo.

Comisión Nacional para Prevenir y Erradicar la Violencia contra las Mujeres: CONAVIM. (2009). Diagnóstico sobre la realidad social, económica y cultural de los entornos locales para el diseño de intervenciones en materia de prevención y erradicación de la violencia en la región sur: el caso de la zona metropolitana de Mérida, Yucatán. http://cedoc.inmujeres. gob.mx/lgamvlv/conavim/merida.pdf

Dirección General de Epidemiologia. (2020). COVID-19 México. https://coronavirus.gob.mx/datos/
Dirección General de Planeación, Programación y Estadística Educativa: DGPPyEE (2019). Principales cifras 2018-2019. https://www.planeacion.sep.gob.mx/Doc/ estadistica e indicadores/principales cifras/ principales cifras 2018 2019.pdf

Federación Internacional de Trabajadores Sociales: FITS. (2018). Declaración global de Trabajo Social de principios éticos. https://www.ifsw.org/global-social-workstatement-of-ethical-principles/

Fondo de las Naciones Unidas para la Infancia: UNICEF (2015). Una aproximación a la situación de adolescentes y jóvenes en América Latina y el Caribe a partir de evidencia cuantitativa reciente. https://www.unicef.org/lac/sites/unicef. org.lac/files/2018-04/UNICEF Situacion de Adolescentes y Jovenes en LAC junio2105.pdf

Galeana, S. (2005). Campos de acción del Trabajo Social. En Sánchez, M. Manual de Trabajo Social. Plaza y Valdés.

García, C; y Ruiz, O. (2011). La segregación territorial y el rezago en el sur de la ciudad de Mérida, como el resultado del crecimiento urbano descontrolado. Quivera; Revista de Estudios Territoriales, 13(1),122-138. 
García, G; Oliva, Y; y Ortiz, R. (2012). Distribución espacial de la marginación urbana en la ciudad de Mérida, Yucatán, México. Investigaciones Geográficas, Boletín del Instituto de Geografía, UNAM, 77 (1), 89,106 .

Instituto Nacional de Estadística y Geografía de México: INEGI (2015). Encuesta Intercensal 2015. https://www.inegi.org.mx/temas/ estructura/default.htm|\#Informacion general

Instituto Nacional para la Evaluación de la Educación: INEE. (2018). La permanencia, principal problema en la EMS: docentes. https://www. inee.edu.mx/la-permanencia-principalproblema-en-la-ems-docentes/

Jara, O. (2013). La sistematización de experiencias: práctica y teoría para otros mundos posibles. Centro de Estudios y Publicaciones Alforja.

Ley General de Educación (2019).

http://www. diputados.gob.mx/LeyesBiblio/ pdf/LGE 300919.pdf

Organización de las Naciones Unidas: ONU. (2019). Juventud. https://www.un.org/es/sections/issues-depth/ youth-0/index

html\#: :text=No\%20existe\%20una\%20 definici\%C3\%B3n\%20internacional,entre\%20 15\%20y\%2024\%20a\%C3\%B1os.
Organización Mundial de la Salud: OMS (2020a). Salud de los adolescentes. https://www.who.int/ topics/adolescent health/es/

Organización Mundial de la Salud: OMS. (2020b). Preguntas y respuestas sobre la enfermedad por coronavirus: COVID-19. https:// www.who.int/es/emergencies/diseases/ novel-coronavirus-2019/advice-for-public/q-acoronaviruses

Salcedo, E. (2020). Coronavirus: ¿Qué le toca hacer al trabajo social para enfrentar la pandemia del siglo XXI? Nueva acción crítica. Diálogos desde el Trabajo Social Latinoamericano, 7, 88-94.

Unidad Académica Bachillerato con Interacción Comunitaria: UABIC. (S.F.). Historia. http://www. unidadacademica.uady.mx/historia.php

Varea, E. (2020). Emergencias sociales ante el COVID-19. http://www.cgtrabajosocial.es/app/webroot/ files/consejo/files/emergencias/COVID19\%20 22\%20ABRIL\%201 compressed.pdf

Vázquez, D. (2008). Problemas más frecuentes en estudiantes de bachillerato (Tesis de Maestría). Universidad Autónoma de Yucatán. México. 


\section{Notas}

1. La Universidad Autónoma de Yucatán, oferta tres programas educativos de bachillerato, que consta de la Escuela Preparatoria Número Uno, Escuela Preparatoria Número Dos, y la Unidad Académica Bachillerato con Interacción Comunitaria. 